\title{
CORRECTIONS
}

\section{Pharmacological prevention of migraine}

Clarification-In this Clinical Review (BMJ 2011;342:d583, doi:10.1136/bmj.d583), doses of drugs for migraine prevention were presented in a table with the abbreviation "QD," meaning "once daily," as defined in the table footnote. To avoid prescribing an overdose, readers should not confuse "QD" with
"QDS," which means "four times a day." The $B M J$ will avoid using these acronyms wherever possible.

Cite this as: BMJ 2013;346:f507

๑ BMJ Publishing Group Ltd 2013 